\title{
Politetrafluoroetileno e esclera fumana no tratamento cirúrgico de perfuração escleral em coelfios
}

\author{
Polytetrafluoroethylene and fuman sclera in surgical treatment of scleral perforation \\ in rabbits
}

$\begin{array}{lcr}\text { Simone } & \text { Pezzutti } & \\ \text { Nilo } & \text { Holzchufi } & \\ \text { Milton } & \text { Ruiz Alves }{ }^{3} & \\ \text { Marlene } & \text { Pezzutti } & \text { Holzchufh } \\ \text { Ricardo } & \text { Holzchufh } & \end{array}$

\section{RESUMO}

Objetivo: Foi realizado estudo experimental para avaliar o comportamento do politetrafluoroetileno $\left(\right.$ Gore-Tex $\left.{ }^{\circledR}\right)$ em relação à esclera humana, em perfurações esclerais produzidas em olhos de coelhos. Métodos: Vinte e dois olhos de coelhos foram submetidos à perfuração escleral seguida da colocação e sutura do enxerto de Gore-Tex ${ }^{\circledR}$ no olho esquerdo e esclera humana no olho direito. A evolução pós-operatória foi avaliada diariamente durante um mês e, analisada a intensidade da hiperemia ocular, presença de infecção, secreção ocular, rejeição e tonicidade do olho à digito-pressão. Resultados: Não foi observada presença de secreção, casos de infecção ou rejeição. As secções histopatológicas mostraram presença de processo inflamatório não granulomatoso tipo fibrose nos olhos com Gore-Tex ${ }^{\circledR}$, com boa adesão e epitelização. Conclusão: $O$ Gore-Tex ${ }^{\circledR}$ mostrou ser material plausível quando utilizado nos defeitos e perfurações esclerais com algumas vantagens como fácil obtenção, manuseio e durabilidade.

Descritores: Esclera/transplante; Politetrafluoroetileno; Esclera/cirurgia; Esclera/patologia; Coelhos

INTRODUÇÃO

Como todo componente do olho, a esclera pode ser sítio de afecções puramente oculares ou refletir manifestações de doenças sistêmicas ${ }^{(1)}$. Isto é patente nos casos de inflamações com ou sem infecções (esclerites) ${ }^{(2-3)}$, queimaduras, traumatismos e complicações pós-operatórias (facectomia, exerese de pterígio ${ }^{(4-6)}, \beta$-terapia e cerclagem de retina ${ }^{(1,7-8)}$. Entre as principais doenças, que levam ao afilamento escleral e corneo-escleral estão escleromalácia perfurante e as esclerites necrosantes. Em nosso meio uma das causas principais de afinamento escleral e corneo-escleral ocorre tardiamente após cirurgia de pterígio, seguida de aplicação de $\beta$-terapia ${ }^{(5)}$.

A abordagem cirúrgica é habitual nos defeitos esclerais ${ }^{(2-3,9)}$, obtendose bons resultados com o uso de vários materiais para enxertia, que podem ser autólogos, homólogos e sintéticos ${ }^{(7)}$. A esclera homóloga permanece como substituto natural mais utilizado nos procedimentos tectônicos ${ }^{(10-11)}$. As principais vantagens do seu emprego são: facilidade de obtenção, elasticidade, composição da estrutura de colágeno, proporcionando bom suporte para o bulbo ocular e ausência de componentes celulares ativos. Esta última característica evita reações imunológicas, e favorece o pronto uso da esclera logo após a remoção ${ }^{(3)}$. A terapêutica cirúrgica para os defeitos esclerais atualmente conta com emprego de materiais sintéticos, estando em uso o politetrafluoroetileno ${ }^{(7,12-15)}$. 
O politetrafluoroetileno foi desenvolvido por W.L. Gore e associados, na década de 1960. É manufaturado em tiras de vários comprimentos, com espaços internodais de diferentes tamanhos $^{(12)}$. Na década de 1970, foi utilizado experimentalmente pela primeira vez como substituto vascular em cães e, logo após, em seres humanos ${ }^{(12-14,16)}$.

$\mathrm{Na}$ Oftalmologia, a utilização desse material é recente ${ }^{(14-16)}$. Em 1989, Tawakol et al..$^{(17)}$ realizaram estudo preliminar histológico do implante de politetrafluoroetileno em esclera de coelhos, durante seis semanas. Concluíram que o material era de fácil manuseio, apresentou boa adaptação à esclera e não provocou reação inflamatória, mas verificaram que não houve adesão do material à esclera e aos tecidos vizinhos.

Whitmore et al, em $1990^{(18)}$, realizaram estudo piloto na esclera de 12 coelhos, a fim de verificar a aplicação de GoreTex $^{\circledR}$, Miragel ${ }^{\circledR}$ e Dacron ${ }^{\circledR}$ como reforço escleral no tratamento da miopia em seres humanos. Todos os materiais acima testados foram biocompatíveis, sendo os dois primeiros resistentes à invasão por tecido fibrovascular.

Em 1991, Legeais et al. ${ }^{(8)}$ trataram com sucesso seis pacientes com perfurações corneais e dois com perfurações paralímbicas, utilizando enxerto temporário de politetrafluoroetileno até o definitivo transplante de córnea. Os autores concluíram que o Gore-Tex ${ }^{\circledast}$ parece ser seguro e efetivo para ser usado no tratamento de urgências de perfurações corneais centrais e periféricas. Por outro lado, Huang et al. (1994) $)^{(4)}$ aplicaram enxerto de politetrafluoroetileno em três pacientes com defeitos de córnea ou esclera.

No exame histopatológico não foi evidenciada epitelização do enxerto, nenhuma reação celular e ausência de crescimento fibrovascular. Embora esse enxerto ofereça algumas vantagens, os autores mostraram desvantagens quando é usada sem adequado recobrimento conjuntival, incluindo pobre epitelização, fraca adesão entre o enxerto e os tecidos vizinhos e potencial rota para infecções.

Este estudo foi realizado com objetivo de avaliar o uso de politetrafluoroetileno como material alternativo à esclera no tratamento de perfuração escleral em coelho.

\section{METODOS}

Foram utilizados 22 olhos de onze coelhos albinos da raça Nova Zelândia, adultos, machos, sadios, com peso médio de $2.070 \mathrm{~kg} \pm 0.4$, alojados no biotério da Técnica Cirúrgica da Santa Casa de Misericórdia de São Paulo.

Os materiais usados foram Esclera humana (proveniente do Banco de Olhos da Santa Casa da Misericórdia de São Paulo) e Politetrafluoroetileno (Gore-Tex ${ }^{\circledR}$ - 160-04-High Porosity PTFE Tubing, doado pela International Polymer Engineering).

Todos os animais do experimento foram submetidos ao mesmo protocolo anestésico. A tranqüilização foi realizada com mistura de fentanil-droperidol (Inoval) na dose de $0,3 \mathrm{cc} /$ $\mathrm{kg}$ por via intramuscular e após 10 minutos foi feita indução anestésica com associação de tiletamina e zolazepam (Zoletil-
50) na dose de $0,4 \mathrm{cc} / \mathrm{kg}$ de peso vivo. Na manutenção da anestesia foi empregado o agente inalatório halotano.

Logo após a antissepsia com polvidine tópico, foi realizado em todos os coelhos o seguinte procedimento cirúrgico: colocação de blefarostato no olho a ser operado; peritomia conjuntival de $6 \mathrm{~mm}$ na região temporal, na posição de $9 \mathrm{~h}$, próxima do limbo; perfuração da esclera de $2 \mathrm{~mm} \times 2 \mathrm{~mm}$ realizada com lâmina de bisturi $\mathrm{n}^{\circ} 11$; preparação de enxerto escleral e de politetrafluoroetileno (Gore-Tex ${ }^{\circledR}$ ) de $3 \mathrm{~mm}$ X $3 \mathrm{~mm}$; colocação no olho direito de enxerto de esclera humana; colocação no olho esquerdo de enxerto de politetrafluoroetileno; sutura dos enxertos sobre a perfuração escleral com dois pontos simples, realizados com o fio Vicryl 6-0 ${ }^{\circledR}$ nas duas extremidades verticais dos enxertos, transfixando os $2 / 3$ anteriores da esclera; recobrimento conjuntival suturado com ponto simples e injeção subconjuntival de $1 \mathrm{cc}$ da mistura de uma ampola de $1 \mathrm{cc}$ de gentamicina base de $40 \mathrm{mg}$ e 1 ampola de $2,5 \mathrm{cc}$ de fosfato dissódico de dexametasona. Durante a primeira semana pós-operatória, foi instilada uma gota de colírio de tobramicina ( $3 \mathrm{mg}$ ) com dexametasona (1 mg), três vezes ao dia, em ambos os olhos dos coelhos. A evolução pós-operatória foi analisada diariamente, no período de um mês, e a cada avaliação, atribuíram-se notas que traduziam a intensidade da hiperemia ocular ( 0 ausência; + hiperemia leve; ++ hiperemia moderada; +++ hiperemia intensa) e a presença ou ausência de secreção ocular. Avaliou-se também a tonicidade do olho à digito-pressão, durante a primeira semana.

No $7^{\circ}$ e $15^{\circ}$ dia após a operação foram enucleados 6 olhos de 3 coelhos ( 6 coelhos no total) e no $30^{\circ}$ dia, 10 olhos de 5 coelhos. Estes olhos foram conservados em solução de formaldeído a $10 \%$ e encaminhados para processamento histopatológico com objetivo de observar a presença ou não de processo inflamatório, o tipo de inflamação (granulomatosa ou não granulomatosa) e sua intensidade evolutiva (aguda, crônica e fibrose).

Para análise dos resultados relativos a hiperemia ocular causada pelos dois materiais (Gore-Tex ${ }^{\circledast}$ e esclera humana), foi aplicado teste estatístico através de provas não paramétricas (análise de variância - ANOVA, seguida do teste de Friedman). O grau de significância estabelecido foi de $5 \%(p<0,05)$.

\section{RESULTADOS}

$\mathrm{Na}$ análise estatística dos graus da hiperemia ocular nos olhos de coelhos com enxerto de Gore-Tex ${ }^{\circledR}$ e esclera humana isoladamente (Quadros 1 e 2), não foi observada diferença significativa $(p>0,05)$. Mas quando comparados entre si evidenciou-se diferença significante $(\mathrm{p}<0,05)$ referentes à ausência de hiperemia (grau 0) (Gráfico 1).

Durante o estudo, não foi evidenciada presença de secreção ou de sinais de infecção. Não houve diferença quanto à tonicidade nos grupos tratados com Gore-Tex ${ }^{\circledR}$ e esclera humana. Pela observação clínica, os enxertos apresentavam boa obliteração à perfuração. 


\begin{tabular}{|c|c|c|c|c|c|c|c|c|}
\hline \multicolumn{9}{|c|}{$\begin{array}{l}\text { Quadro 1. Avaliação diária da hiperemia conjuntival nos olhos de } \\
\text { coelhos com enxerto de Gore-Tex }{ }^{\circledR} \text {. Santa Casa de Misericórdia de } \\
\text { São Paulo, SP, } 1998\end{array}$} \\
\hline \multicolumn{9}{|c|}{ GRAU DE HIPEREMIA } \\
\hline \multirow[b]{2}{*}{ Dias } & \multicolumn{2}{|l|}{+++} & \multicolumn{2}{|l|}{++} & \multirow{2}{*}{$\begin{array}{c}+ \\
n^{\circ} \\
\text { olhos }\end{array}$} & \multicolumn{3}{|c|}{0} \\
\hline & $\begin{array}{c}n^{\circ} \\
\text { olhos }\end{array}$ & $\%$ & $\begin{array}{c}n^{\circ} \\
\text { olhos }\end{array}$ & $\%$ & & $\%$ & $\begin{array}{c}n^{\circ} \\
\text { olhos }\end{array}$ & $\%$ \\
\hline $1^{\circ}$ & 11 & 100 & 0 & 0 & 0 & 0 & 0 & 0 \\
\hline $2^{\circ}$ & 2 & 18,18 & 9 & 81,82 & 0 & 0 & 0 & 0 \\
\hline $3^{\circ}$ & 0 & 0 & 10 & 90,91 & 1 & 9,09 & 0 & 0 \\
\hline $4^{\circ}$ & 0 & 0 & 2 & 18,18 & 9 & 81,82 & 0 & 0 \\
\hline $5^{\circ}$ & 0 & 0 & 0 & 0 & 9 & 81,82 & 2 & 18,18 \\
\hline $6^{\circ}$ & 0 & 0 & 0 & 0 & 1 & 9,09 & 10 & 90,91 \\
\hline \multirow[t]{2}{*}{$7^{\circ}$} & 0 & 0 & 0 & 0 & 0 & 0 & 11 & 100 \\
\hline & \multicolumn{4}{|c|}{ +++ hiperemia severa } & \multicolumn{3}{|c|}{ + hiperemia leve } & \\
\hline
\end{tabular}

\begin{tabular}{|c|c|c|c|c|c|c|c|c|}
\hline \multicolumn{9}{|c|}{$\begin{array}{l}\text { Quadro 2. Avaliação diária da hiperemia conjuntival nos olhos de } \\
\text { coelhos com enxerto de esclera humana. Santa Casa de Misericórdia } \\
\text { de São Paulo, SP, } 1998\end{array}$} \\
\hline \multirow[b]{3}{*}{ Dias } & \multicolumn{8}{|c|}{ GRAU DE HIPEREMIA } \\
\hline & \multicolumn{2}{|l|}{+++} & \multicolumn{2}{|l|}{++} & \multirow{2}{*}{$\begin{array}{c}+ \\
n^{\circ} \\
\text { olhos }\end{array}$} & \multicolumn{3}{|c|}{0} \\
\hline & $\begin{array}{l}n^{\circ} \\
\text { olhos }\end{array}$ & $\%$ & $\begin{array}{l}n^{\circ} \\
\text { olhos }\end{array}$ & $\%$ & & $\%$ & $\begin{array}{l}n^{\circ} \\
\text { olhos }\end{array}$ & $\%$ \\
\hline $1^{\circ}$ & 11 & 100 & 0 & 0 & 0 & 0 & 0 & 0 \\
\hline $2^{\circ}$ & 9 & 81,82 & 2 & 18,18 & 0 & 0 & 0 & 0 \\
\hline $3^{\circ}$ & 4 & 36,36 & 7 & 63,64 & 0 & 0 & 0 & 0 \\
\hline $4^{\circ}$ & 0 & 0 & 10 & 90,91 & 1 & 9,09 & 0 & 0 \\
\hline $5^{\circ}$ & 0 & 0 & 6 & 54,55 & 5 & 45,45 & 0 & 0 \\
\hline $6^{\circ}$ & 0 & 0 & 2 & 18,18 & 7 & 63,64 & 2 & 18,18 \\
\hline \multirow[t]{2}{*}{$7^{\circ}$} & 0 & 0 & 0 & 0 & 5 & 45,45 & 6 & 54,55 \\
\hline & \multicolumn{4}{|c|}{$\begin{array}{l}\text { +++ hiperemia severa } \\
++ \text { hiperemia moderada }\end{array}$} & \multicolumn{4}{|c|}{$\begin{array}{l}\text { + hiperemia leve } \\
0 \text { ausência de hiperemia }\end{array}$} \\
\hline
\end{tabular}

Durante análise dos cortes histopatológicos, em quatro olhos foi observada presença de processo inflamatório granulomatoso, tipo corpo estranho, que foi relacionado ao fio de sutura, sendo indiferente nos olhos com enxerto de Gore-Tex ${ }^{\circledast}$ ou esclera humana.

\section{CONCLUSÕES}

Neste estudo a análise estatística comparativa entre a ocorrência de hiperemia ocular nos olhos de coelhos com enxerto de Gore-Tex ${ }^{\circledR}$ e com esclera humana evidenciou-se diferença significante $(p<0,05)$ nos resultados referentes à ausência de hiperemia (grau 0). Esta diferença foi resultado de ocorrência de reação inflamatória aguda nestes olhos, que mais tarde evoluíram para o desenvolvimento de fibrose.

Não foram observados secreção ocular e sinais de infecção ou extrusão dos enxertos de Gore-Tex ${ }^{\circledR}$ ou de esclera humana ratificando o bom desempenho desses tecidos como opção de reforço escleral. Esses achados coincidem com o trabalho de Legeais et al. ${ }^{(8)}$, no qual também não foi constata-

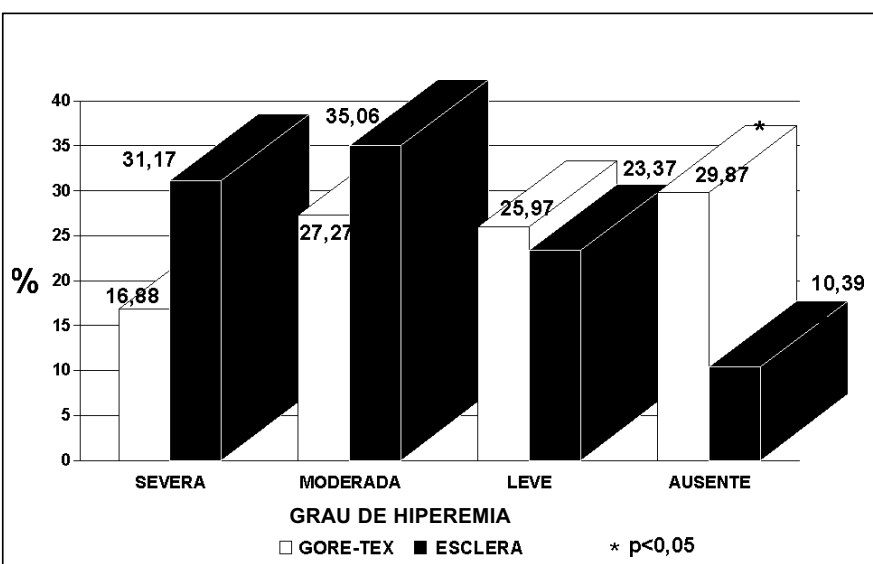

Fonte: Santa Casa de Misericórdia de São Paulo. São Paulo, 1998.

Gráfico 1 - Média da porcentagem dos graus da hiperemia ocular nos olhos de coelhos com enxerto de Gore-Tex ${ }^{\circledR}$ e esclera humana

do nenhum sinal de infecção ou rejeição nos olhos tratados com Gore-Tex ${ }^{\circledR}$; mas diferem das conclusões de Huang et al. ${ }^{(4)}$ que relacionaram a presença de um caso de infecção (endoftalmite) entre os seus pacientes ao uso de Gore-Tex ${ }^{\circledast}$ como enxerto corneo-escleral, responsabilizando o material como provável rota de infecção.

A tonicidade ocular foi verificada apenas pela digitopressão, sem emprego de aparelhos, e teve o simples objetivo de avaliar, de modo grosseiro, a obliteração da perfuração escleral. Isto foi observado na primeira semana de pós-operatório, durante a instilação do colírio de antibiótico, e não houve diferença entre os olhos de enxerto de esclera humana $\mathrm{e}$ Gore-Tex ${ }^{\circledR}$.

As secções histopatológicas evidenciaram que todos os olhos com enxerto de Gore-Tex ${ }^{\circledR}$ apresentavam processo inflamatório, enquanto esse processo foi observado em apenas 63 , $64 \%$ ( 7 olhos) com enxerto de esclera (Gráfico 2).

Dos 11 olhos de coelhos enxertados com Gore-Tex ${ }^{\circledR}$, em nove $(81,82 \%)$ foi evidenciado processo inflamatório, que evoluiu para fibrose. (Tabela 1 e Gráfico 3) Este fato foi indiferente aos dias em que os olhos foram enucleados $\left(7^{\circ}, 15^{\circ}\right.$ e $30^{\circ}$ dias). $\mathrm{O}$ estudo histopatológico com enxerto de esclera humana mostrou que $54,55 \%$ ( 6 olhos) tinham processo inflamatório crônico, 9,09\% (1 olho) fibrose e 36,36\% (4 olhos) não apresentavam processo inflamatório. Dos 4 olhos que não apresentaram processo inflamatório, 3 haviam sido enucleados no $7^{\circ}$ dia. (Tabela 1 e Gráfico 3 )

A reação fibrótica observada nesses olhos tem provável origem no manuseio cirúrgico e na presença de tecido fibroso vascular do próprio recobrimento conjuntival na área do enxerto. A área fibrótica não penetrou o material, mas atuou como arcabouço mecânico de reforço da região.

Neste estudo foi possível constatar que o enxerto escleral com Gore-Tex ${ }^{\circledR}$ é de fácil manuseio, flexível, maleável, com boa adaptação e obliteração à perfuração escleral, concordando 


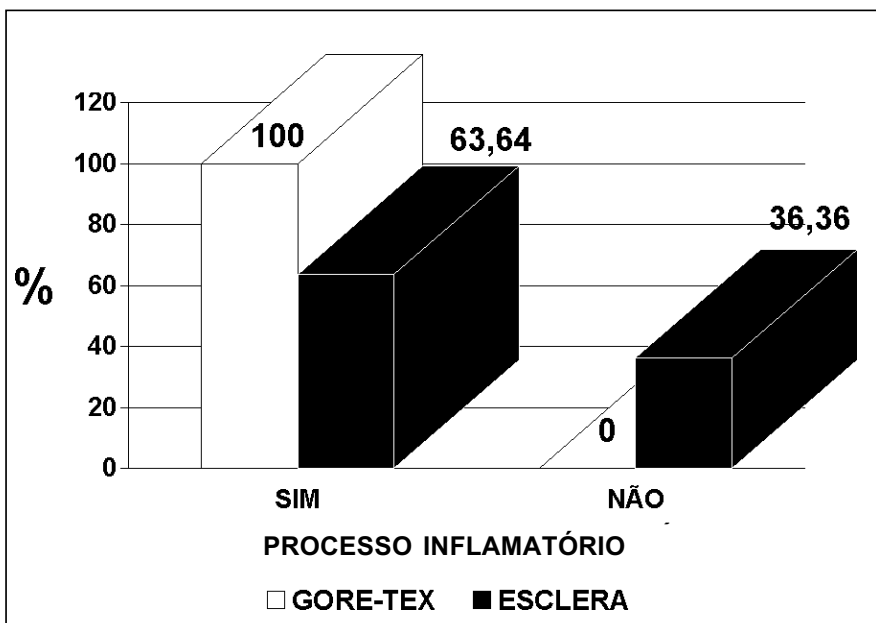

Fonte: Santa Casa de Misericórdia de São Paulo. São Paulo, 1998.

Gráfico 2 - Porcentagem de presença de processo inflamatório nos olhos de coelhos com enxerto de Gore-Tex ${ }^{\circledR}$ e esclera humana, avaliada histopatologicamente

\begin{tabular}{|c|c|c|c|c|}
\hline & PRO & sso II & AMATÓRIO & \\
\hline & GORE & & ESCLERA HL & MANA \\
\hline & $n^{0}$ de olhos & $\%$ & $n^{\circ}$ de olhos & $\%$ \\
\hline Ausente & 0 & 0 & 4 & 36,36 \\
\hline Inicial & 2 & 18,18 & 0 & 0 \\
\hline Crônico & 0 & 0 & 6 & 54,55 \\
\hline Fibrose & 9 & 81,82 & 1 & 9,09 \\
\hline TOTAL & 11 & 100 & 11 & 100 \\
\hline
\end{tabular}

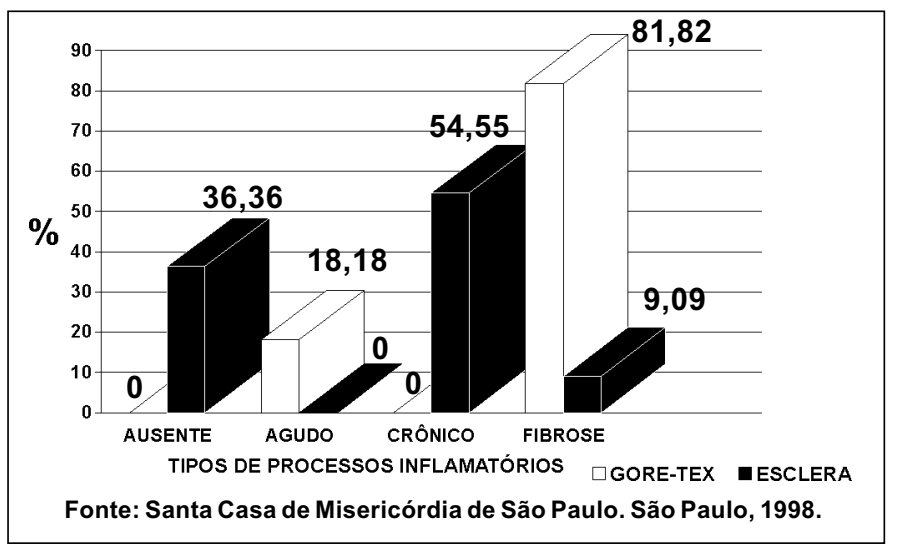

Gráfico 3 - Incidência do tipo de processo inflamatório observado no exame histopatológico, nos olhos de coelhos tratados com enxerto de Gore-Tex $^{\circledR}$ e esclera humana

com Huang et al. ${ }^{(4)}$, Legeais et al. ${ }^{(8)}$, Tawakol et al. ${ }^{(17)}$ e Withmore et al. ${ }^{(18)}$. Já o manuseio da esclera humana, material biológico, apesar de fácil obtenção e de não possuir componentes celulares ativos, por ser fibrosa, é de manuseio mais difícil.
Os resultados evidenciados neste estudo foram inéditos em relação ao uso de Gore-Tex ${ }^{\circledR}$ em Oftalmologia, pois todos os trabalhos publicados com uso desse material sobre a esclera evidenciaram pobre epitelização, com resistência do tecido ao crescimento fibrovascular ${ }^{(12-15)}$, mas provavelmente isso se deve ao fato de ter-se feito o recobrimento conjuntival protegendo a área enxertada. Em resumo o Gore-Tex ${ }^{\circledR}$ ofereceu vantagens para ser usado em defeitos e perfurações, por ser de fácil obtenção, manuseio e durabilidade. Proporcionou boa aderência, serviu de suporte para o crescimento de tecido fibroso e não desencadeou sinais de infecção. Esses fatores o caracterizam como material alternativo.

\section{A B S T RAC T}

Purpose: An experimental study to evaluate the behavior of polytetrafluoroethylene (Gore-Tex ${ }^{\circledR}$ ) compared with human sclera, in scleral perforations induced in rabbits eyes was performed. Methods: Twenty-two eyes of rabbits were submitted to scleral perforation followed by Gore-Tex ${ }^{\circledR}$ graft in the left eye and human sclera graft in the right eye respectively. During one month the postoperative evolution was analyzed every day: intensity of hyperemia, presence of infection, secretion, rejection and tonicity of the eyes. Results: No cases of secretion, infection or rejection were observed. The histological sections showed fibrosis in the eyes with Gore-Tex ${ }^{\circledR}$, good adhesion and epithelization. Conclusion: The Gore-Tex ${ }^{\circledR}$ showed to be a plausible material to be used as graft in scleral defects with some advantages such as easy obtention, good handling and durability.

Keywords: Sclera/transplantation; Polytetrafluoroethylene; Sclera/surgery; Sclera/pathology; Rabbits

\section{REFERENCIAS}

1. Koenig SB, Kaufman HE. The treatment of necrotizing scleritis with an autogenous periosteal graft. Ophthalmic Surg 1983;14:1029-32.

2. Blum Jr. FG, Salamoun SG. Scleromalacia perforans: a useful surgical modification in fascia lata or scleral grafting. Arch Ophthalmol 1963;69:287.

3. Merz EH. Scleral reinforcement with aortic tissue. Am J Ophthalmol 1964; 57:66-70.

4. Huang WJ, Hu FR, Chang S. Clinicopathologic study of gore tex path grafting corneoscleral surgery. Cornea 1994;13:82-6.

5. Mauriello Jr. JA, Pokorny K. Use of split-tickness dermal grafts to repair corneal and scleral defets - a study of 10 patients. Br J Ophtalmol 1993;377: 327-31.

6. Nishiwaki-Dantas MC, Abbott RL, Dantas PEC. Use of corneal patch graft repair scleral defect secondary to pterygium excision and topical mitomycin-C therapy. Arq Bras Oftalmol 1995;58:170-3.

7. Koenig SB, Sanitato JJ, Kaufman HE. Long-term follow-up study of scleroplasty using autogenous periosteum. Cornea 1990;9:139-43.

8. Legeais JM, Renard G, D’Hermies F, Rossi C, Pouliquen Y. Surgical management of corneal perforation with explanded polytetrafluorethylene (Gore Tex). Ophthalmic Surg 1991; 22:213-7.

9. Bick MW. Surgical treatment of scleromalacia perforans. Arch Ophthalmol 1959;61:907-17.

10. Mauriello JR JA, Fiore PM, Pokorny KS, Cinotti DJ. Use of split-tickness dermal grafts in the surgical treatment of corneal and scleral defects. Am J Ophthalmol 1988; 105:244-7. 
11. Nishiwaki-Dantas MC, Abbott RL, Dantas PEC. Reparação de defeito escleral ou corneo-escleral com enxerto corneano. Arq Bras Oftalmol 1995;58:295-8.

12. Florian A,Cohn LH, Dammin GJ, Collins JJ. Small vessel replacement with Gore-Tex (expanded polytetrafluoroethylene). Arch Surg 1976;111:267-70.

13. Kenyon KR, Wagoner MD, Hettinger MT. Conjunctival autograft transplantation for advanced and recurrent pterygium. Ophthalmology 1985;92:1461- 70.

14. Obear MF, Winter FC. Technique of overlay scleral homograft Arch Ophthalmol 1964;71:837-8
15. Raizman MB, Dsainz de la Maza M, Foster CS. Tectonic keratoplasty for peripheral ulcerative keratitis. Cornea 1991;10:312-6.

16. Breslin CW, Katz JI, Kaufman, HE. Surgical treatment of necrotizing scleritis. Arch Ophthalmol 1977;95:2038-40.

17. Tawakol ME, Peyman G, Lui KR, Kaufman HE. Gore-Tex soft tissue bands as scleral explants in rabbits a preliminary histologic study. Ophtthalmic Surg 1989;20:199-201.

18. Whitmore WG, Harrison W, Curtis BJ. Scleral reinforcement in rabbits using synthetic graft materials. Ophthalmic Surg 1990;21:327-30.

\section{CURSO BÁSICO UNIFICADO DE OFTALMOLOGIA TURMA: João Francisco Cêntola Nóbrega - 2002}

\section{4/01/02 a 07/02/02 - Período Integral - 160 horas}

Local: Anfiteatro de Oftalmologia HCFMUSP - Instituto Central - $6^{\circ}$ andar - Sala 6115 Av. Dr. Enéas de Carvalho Aguiar, 255 - Cerqueira César - São Paulo

\section{ORGANIZAÇ̃̃O}

Newton Kara-José

Flávio Fernandes Villela

Regina Kazumi Noma de Campos

Bruno Campelo Leal

Amaryllis Avakian

Emerson Fernandes de Souza e Castro

Liane Touma

Vinícius Paganini Nascimento

\section{COORDENADORES}

Antonio Augusto Velasco Cruz

Carlos Eduardo Leite Arieta

Denise Fornazari Oliveira

Harley Edison do Amaral Bicas

Maria Rosa Bet de Morais

Rosane Silvestre de Castro

Sidney Faria de Souza

Silvana Schellini
- Faculdade de Medicina da Universidade de São Paulo - FMUSP

- Universidade Estadual de Campinas - UNICAMP

- Faculdade de Medicina de Ribeirão Preto - FMUSP

- Universidade Estadual Paulista - UNESP
- Faculdade de Medicina do Triângulo Mineiro

- Serviço de Oftalmologia Instituto "Benjamim Constant"

- Universidade de Taubaté

\section{GRANDE OPORTUNIDADE DA RECICLAGEM (vagas limitadas)}

\section{Inscrição para Curso integral valor: RS 800,00 Inscrições por dia valor: RS 100,00 Informações: Telefone: (011) 3069-6289 - 3069-7217 - Horário comercial}

- Anamnese e Dinâmica do Consultório Oftalmológico

- Anatomia e Fisiologia da Órbita

-Anatomia e Fisiologia: Conjuntiva, Córnea e Esclera

- Anatomia e Fisiologia: Pálpebras e Vias Lacrimais

- Angiofluoresceinografia

- Ângulo da Câmara Anterior, Humor Aquoso, Pressão

Intra-Ocular

- Banco de Olhos - Avaliação

- Biomicroscopia

- Campo Visual

- Catarata de A-Z - Curso Especial

- Cirurgia Estrabismo

- Cirurgia Refrativa

- Conceitos Ópticos do Olho

- Curso de Lentes de Contato

- Doenças Externas

- Ecografia

- Eletrofisiologia

-Embriologia/Genética

- Exames Complementares

- Farmacologia

- Glaucoma

\section{CURSOS}

- Imunologia

- Motilidade Extrínseca

- Motilidade Ocular, Visão Binocular

- Oftalmoscopia Indireta: Teórico Prático

- Olho da Criança e do Idoso

- Óptica

- Prática Oftalmológica

- Pré e Pós Operatório

- Princípios Básicos em Pesquisa Oftalmológica

- Princípios de Laser em Oftalmologia

- Projeto Catarata

- Refração - Curso Especial

- Refração : O exame do Paciente

- Refração: Anomalias do Sistema Óptico do Olho

- Retina Cirúrgica

- Retina Clínica

- Semiologia Neuro-Oftalmológica

- Úrgências Oftalmológicas

- Úvea e Retina

- Uveítes

- Vias Lacrimais/Pálpebras

-Visão Subnormal 